\title{
LA CIVILIZACIÓN DE LOS CUERPOS: LAS PEDAGOGÍAS SEXUALES Y DE GÉNERO EN LA EDUCACIÓN PRIMARIA COSTARRICENSE
}

\section{CIVILIZING THE BODIES: SEX AND GENDER PEDAGOGIES IN COSTA RICAN EDUCATION}

\author{
María Isabel Gamboa Barboza*
}

RESUMEN

Tras un análisis de discurso y con el objeto de estudiar los mecanismos disciplinares de las sexualidades y los géneros existentes en los programas de la educación primaria costarricense, se sugiere que estos son producto de dos tendencias discursivas: una liberal y otra conservadora. A diferencia de los hallazgos de una investigación que se realizó anteriormente, con programas producidos a lo largo de más de cuatro décadas, en la que predominó un tipo de rechazo a la diversidad sexual, en los actuales, el conservadurismo tiene un tinte especialmente misógino.

PALABRAS CLAVE: EDUCACIÓN * COSTA RICA * SEXUALIDAD * GÉNERO * CONSERVADURISMO

ABSTRACT

After a discourse analysis of the disciplinary mechanisms of sex and gender in Costa Rican primary education, I suggest that these are a product of two discursive tendencies: liberal and conservative. Nevertheless, in contrast with the results I found in a previous study of programs which were produced alongside more than four decades, during which a kind of rejection of sexual diversity was predominant, in the present, conservatism has a kind of special misogynous turn.

KEYWORDS: EDUCATION * COSTA RICA * SEXUALITY * GENDER * CONSERVATISM

Escuela de Sociología y Programa de Posgrado en Estudios de la Mujer, Universidad de Costa Rica, Costa Rica. isabelgamboabarboza@gmail.com 


\section{INTRODUCCIÓN}

En el año 2002, se publicó un ensayo de mi autoría producto de una investigación historiográfica en la que se demostró que los libros de texto usados por la educación pública costarricense a partir de la década de los años 60 del siglo xx y hasta la fecha de dicha investigación, reproducían al menos cuatro paradigmas, que por supuesto se sobreponían unos con otros pero que, con fines explicativos y siguiendo cierta lógica cronológica, se enumeraron así: 1) se caracteriza por una aparente omisión del tema; 2) hace énfasis en lo reproductivo-heterosexual; 3) gira alrededor de una cierta racionalidad científica; y 4) un discurso sexológico y de peligro sexual muy marcado (Gamboa, 2010).

Actualmente, con esta nueva investigación se actualiza el estado y el avance del tratamiento que el Ministerio de Educación Pública (MEP) de Costa Rica, le da a la diversidad sexual $y$ de género $y$, por tanto, de manera indirecta, a las personas que están en la educación primaria.

Los programas de estudio, objeto de esta investigación, están constituidos por 19 libros - correspondientes a todos los que el MEP pone a disposición de docentes, estudiantes, madres $y$ padres para que, de forma voluntaria, los usen como contenido y recurso metodológico- de los cuales, 8 hacían referencia a los temas de interés de esta investigación. De ellos, se seleccionaron todas las referencias a las categorías asociadas al tema en estudio y se les aplicó el análisis de discurso a partir de la propuesta de teóricos como van Dijk (2018), dando énfasis a los temas: la semántica local, el estilo, la argumentación, el relato de anécdotas y las estructuras de la conversación.

Así, mediante el análisis de discurso (Keller, 2011), se revisó cada programa buscando qué se decía, sobre quién, en qué términos y con cuáles propósitos, alrededor de los cuerpos, los deseos y los géneros, con el fin de interpretar cuáles paradigmas subyacen.

Asimismo, en esta investigación se presupone que, al decir de Bourdieu, la institución escolar es la más importante en la consolidación de la formación de sentido, que siempre implica la construcción de sujetos que comparten unas mismas categorías o estructuras de pensamiento (Bourdieu, 1999). Para esto, dicha institución usa, entre otros, una especie de violencia simbólica, aquel mecanismo mediante el cual se imponen como legítimas determinadas maneras de ver y estar en el mundo (Bourdieu y Passeron, 1995). Así, el sistema educativo formal se constituye en el principal socializador de las normas de género (Bourdieu y Passeron, 1995).

A resultas de ello, las normas de género - por impuestas y ficticias - pueden producir sentimientos de dolor y humillación en el estudiantado. Por ejemplo, un contenido que arrincona a las niñas a prepararse para ser esposas y madres, tal y como se encontró en este trabajo, no solo limitará su futuro desarrollo profesional y personal, sino que también podrá provocarle sufrimiento, impotencia y frustración.

Como se detallará, los mecanismos civilizatorios que se reflejan en los programas de estudio están fundados en el habitus, aquellos esquemas mentales y corporales que disponen a ciertas elecciones, $y$ que juegan un papel fundamental en la manera en que la gente vive sus experiencias $y$ las interpreta:

Los agentes sociales están dotados de habitus, incorporados a los cuerpos a través de las experiencias acumuladas: estos sistemas de esquemas de percepción, apreciación y acción permiten llevar a cabo actos de conocimiento práctico, basados en la identificación y el reconocimiento de los estímulos condicionales, y convencionales a los que están dispuestos a reaccionar, así como engendrar (Bourdieu, 1999).

Las constantes indicaciones sobre cómo deben ser los niños y cómo deben ser las niñas, en términos de juegos y comportamiento, integran el habitus encontrado en esta investigación. Como asegura el mismo autor, el habitus se mueve dentro del campo, aquellas relaciones de poder históricas objetivas que median la relación entre personas e instituciones:

Por medio de las estructuras cognitivas $y$ motivadoras que pone en juego (y que siempre dependen, en parte, del campo, 
que actúa como campo de fuerzas formadoras, del que es fruto), el habitus contribuye a determinar las cosas que hay o no hay que hacer, las urgencias, etcétera, que desencadenan la acción (Bourdieu, 1999).

Para este trabajo, los campos con mayor protagonismo son el escolar y el familiar. Del mismo modo, se sugiere que en los programas de estudio, la construcción y el refuerzo de determinado habitus esté afectivizado mediante el uso de cuentos y parábolas que transmitan un mensaje claro a los niños y a las niñas sobre cuál es su lugar. Además de los conocimientos $y$ las aptitudes que la escuela puede inculcar a sus estudiantes y que les posibilita desempeñarse laboral y socialmente, las experiencias educativas también buscan, en último término, que las personas participantes aprendan unos modos de ver $y$ reaccionar socialmente como adecuados frente a otros que son representados como impropios.

Ese proceso de instrucción en la observancia de las leyes sociales fue también estudiado por Foucault para quien, a partir del siglo XVIII, se comienzan a dar los actuales mecanismos de disciplinamiento, concentrados en instituciones, tales como: las cárceles, los hospitales y las escuelas. Consisten básicamente en dispositivos de vigilancia ocultada (ver de forma permanente, sin ser visto) que terminan en la premiación o en el castigo (Foucault, 2002).

$\mathrm{Su}$ fin principal es la modificación de las personas consideradas desviadas, a partir del establecimiento de un orden maniqueo, según el cual las personas y las cosas son buenas o malas. Del mismo modo, Laqueur demuestra el protagonismo del sistema educativo en la regulación de la sexualidad, en particular de la masturbación, a partir del siglo xviII. Desde esa época, los médicos convirtieron a las personas escolares en el paradigma de un personaje: el masturbador. A estos, según Laqueur (2007), sus familias y docentes les vigilaban, castigaban e imponía mecanismos correctivos constantemente.

Desde una importante crítica al ordenamiento sexual y de género, tanto Butler (2011) como Preciado (2013) argumentan que no hay originales, ni de cuerpo, ni de género, ni de deseo; en ese sentido, que no hay una heterosexualidad, ni feminidad, ni masculinidad, original, sino un constante performance que, sea cual sea, debiera poder tener una existencia simbólica. Precisamente, si hay o no originales, y en caso de haber, las múltiples versiones tienen lugar en la vida de la gente, es lo que se pone en juego en los programas de estudios del MEP en los que saltan a la vista los extremos.

Como se demostrará a lo largo de tres apartados, en los programas del MEP se presenta, por un lado, un discurso marcadamente liberal $y$ hasta posmoderno, con respecto no solo al cuerpo sino al deseo y a la concepción genérica de sí; $y$, por otro lado, una posición fuertemente conservadora sobre el cuerpo y su disciplinamiento en dos géneros binarios totalmente definidos, que no deja intersticios para la variedad; junto a ellos, ciertos matices o contracciones.

\section{LA LIBERTAD POSMODERNA}

Las mujeres, $y$ el feminismo en particular, ejercieron una fuerte presión a lo largo de la modernidad para ser consideradas como sujetas de las reivindicaciones y libertades que dicha época marcó (Guzmán y Bonan, 2007). Para Flórez-Estrada (2009), la eclosión de la modernidad hizo posible un salto epistemológico que facilita el pasaje del "reino de la necesidad al reino de la libertad" en los ámbitos culturales y subjetivos.

Efectivamente, ello ha venido generando avances significativos que, sin embargo, no se han traducido en la desaparición de importantes nudos de discriminación hacia las mujeres.

Para el caso de los programas analizados que se han catalogado con contenido liberal, moderno o, incluso, posmoderno, se advierte que el centro de dicha libertad no son las mujeres, sino más bien la diversidad sexual y de género en unas vertientes que se detallarán.

Con lo anterior, no se le está restando importancia al hecho de que este tipo de materiales sean un vínculo a favor de los derechos humanos y del ejercicio de la ciudadanía sexual de la gente, sino que se desea llamar la atención sobre el dato concreto de que las mujeres están 
prácticamente ausentes y que, como se demostrará, aparecen justamente para aplicárseles reglas conservadoras de comportamiento.

Se puede afirmar lo anterior pese a la presencia de ciertos llamados a la igualdad presentes, por ejemplo, en el programa llamado "Estudio de educación para la Vida Cotidiana" 1 que hace mención a la incorporación de "saberes" (Ministerio de Educación Pública, 2017a) relacionados con los derechos humanos y la igualdad de género, en el cual se alude a la diversidad de los seres humanos, incluyendo las condiciones étnicas y de género; además, considera el sexo como una posible causa de exclusión que ocasionaría tristeza, depresión y aislamiento.

A pesar de que se trata de un texto, va más allá y contiene una indicación para que en el aula se promueva la discusión acerca de las creencias sobre las actividades sociales que pueden hacer los hombres y las mujeres, con lo cual se promueve una discusión encaminada hacia "que hay igualdad de capacidades y por lo tanto de oportunidades para hombre y mujeres" (Ministerio de Educación Pública, 2017a, p. 65). A lo anterior, se suma el análisis de los artículos 1, 2 y 7 de la Declaración Universal de los Derechos Humanos que propician la equidad de género, la diversidad y el respeto a las etnias.

Por el momento, se continúa con la línea del discurso liberal comenzando con el programa de "Educación Especial"2, el cual contiene una aclaración que no se puede tomar a la ligera: "Este documento utiliza un lenguaje inclusivo en el formato escrito $y$ responde a procesos de participación activa de hombres $y$ mujeres en equidad" (Ministerio de Educación Pública, 2014, p.7). Lo que esa cita dice es que el programa está asegurado: declara que fue hecho por mujeres y hombres, lo que supondría

1 La educación primaria en Costa Rica se denomina Educación General Básica y se divide en Primer Ciclo, que corresponde al primer y segundo año de estudio; $y$ en Segundo Ciclo, que incluye el tercer, cuarto, quinto y sexto año. El Programa Educación para la vida cotidiana es para el Primer y Segundo Ciclo.

2 Plan de estudios Centro Nacional de Educación Helen Keller (Ministerio de Educación Pública, 2014). en sí mismo garantía de igualdad, al mismo tiempo, utiliza lenguaje inclusivo, que se ofrece como prueba de lo anterior. Este hecho es muy relevante si se considera que el lenguaje inclusivo es uno de los principales campos de batalla, como lo demuestran estudios como los de Yadira Calvo (2017), donde se pelean las luchas por la igualdad $y$ se reciben los ataques de quienes tratan de minimizar y ridiculizar la importancia de la lengua y la manera en que se nombra la existencia.

En tanto, el programa de Inglés ${ }^{3}$ es aún más directo al establecer el respeto como una obligación: "Practice democratic principles such as freedom of expression and religion, respect for plurality and cultural diversity (sexual, linguistic, and ethnic) as stated in the Costa Rican Constitution" (Ministerio de Educación Pública, 2016, p.13).

Ahora bien, uno de los programas se destaca por tener los contenidos más posmodernos, el de Orientación ${ }^{5}$, debido a su llamado a valorar las relaciones de amistad en respeto e igualdad, a reconocer las características físicas $y$ emocionales, al respeto a la diversidad, a identificar los mitos, prejuicios y estereotipos asociados a la sexualidad $y$ al placer $y$ a su enfoque de la sexualidad como algo integral (Ministerio de Educación Pública, 2017b).

Asimismo, el programa se refiere al abuso y al acoso sexual, al bullying y se detiene en la presentación de varias definiciones liberares, tales como, la de ciudadanía sexual, entendida como el poder de tener voz $y$ voto en las políticas y en los programas que afectan la sexualidad, en el ejercicio autónomo de esta; así como a vivir en un Estado que respete y proteja los derechos humanos, el cual se encargará de hacerlos cumplir (Ministerio de Educación Pública, 2017b). En otra

\footnotetext{
3 Segundo Ciclo.

4 Practique principios democráticos como la libertad de expresión y religión, el respeto a la pluralidad y la diversidad cultural (sexual, lingüística y étnica) como se establece en la Constitución de Costa Rica (Traducción propia).

$5 \quad$ Primero y Segundo Ciclos.
} 
de sus definiciones describe las identidades de género como la:

Vivencia interna e individual del género tal como cada persona la siente profundamente, la cual podría corresponder o no con el sexo asignado al momento del nacimiento, incluyendo la vivencia personal del cuerpo (que podría involucrar la modificación de la apariencia o la función corporal a través de medios médicos, quirúrgicos o de otra índole, siempre que la misma sea libremente escogida) $y$ otras expresiones de género, incluyendo la vestimenta, el modo de hablar y los modales (Ministerio de Educación Pública, 2017b, p.132).

Dicha definición es un claro ejemplo de una posición decididamente posmoderna, pues trasciende el tema hasta la modificación, a voluntad, del propio cuerpo.

Además, la apertura en el terreno de los géneros y de las sexualidades se nota en su reconocimiento de la equidad, la diversidad y la no discriminación, de manera muy específica, como en la definición de expresiones de géneros, comprendida como aquellas: "formas en la que una persona expresa su identidad de género, a través de su apariencia física (ropa, cabello, accesorios, etc.), gestos, modos de hablar $y$ patrones de comportamiento" (Ministerio de Educación Pública, 2017b, p. 130). Además, se evidencia en el concepto de orientación sexual comprendido como la capacidad de que una persona tenga atracción y mantenga relaciones emocional, afectiva y sexualmente por personas de su mismo género, de uno diferente o de más de un género (Ministerio de Educación Pública, 2017b), con el cual no solo se desafía el maniqueísmo de género heterosexual, sino el mandato de elegir una sola opción y se abre a una plasticidad circulante de deseos y experiencias.

Este tipo de definiciones no solo representan ideas muy liberales, sino que se adelantaron a una importante resolución internacional conocida como la Opinión consultiva de la Corte Interamericana de Derechos Humanos sobre identidad de género, e igualdad y no discriminación a parejas del mismo sexo
(Corte Interamericana de Derechos Humanos, 2017) que hizo posible que, para el año 2020, las parejas del mismo sexo puedan casarse. Además, ocasionó, como contrarreacción, que el entonces candidato presidencial, Fabricio Alvarado, del partido evangélico Restauración Nacional, estuviera cerca de ganar la presidencia de la República, tal y como está ocurriendo en algunos países de América Latina.

Se trata de un documento adelantado frente a la Clasificación Internacional de Enfermedades (CIE), en la que se persiste en patologizar la disidencia sexual y de género, como se demostró en una investigación previa (Gamboa, 2009). La CIE actual, por ejemplo, clasifica la fluidez de género como una "Discordancia de género" (Clasificación Internacional de Enfermedades, 2019).

Para terminar este apartado, se retoma la ausencia de atención sobre los nudos de daño contra las mujeres porque se trata de una omisión fundamental, pues quiere decir que ni aún en los programas más progresistas se mencionan el sexismo, la violencia sexual, la discriminación salarial ni la subrepresentación de las mujeres en puestos de poder, como lo establecen varias investigaciones e informes, tales como, el Informe Nacional de Desarrollo Humano (Programa de Naciones Unidas para el Desarrollo-Pnud, 2019) y las investigaciones de Mesa (2019), Flores y Espejel (2015), entre muchos otros.

\section{UN VIEJO DISCURSO}

La investigadora María Flórez-Estrada (2019), en un trabajo histórico-sociológico que realizó mediante el análisis de un total de 199 ediciones de 24 periódicos publicados en la prensa escrita costarricense entre 1833-1930, estableció algunas tipologías sobre lo femenino $y$ lo masculino, que fueron promocionadas por los hombres liberales, católicos y comunistas de esa época. Descubrió que, en dicho ámbito, había mayor coincidencia cuando se trataba de los discursos que buscaban modelar a las mujeres (Flórez-Estrada, 2019). Algo semejante se ha encontrado en esta investigación contemporánea, en la que el conservadurismo 
$y$ la misoginia contra las mujeres aparece fuertemente articulado.

Se desea recalcar de primero un programa - el de Estudio de Educación para la Vida Cotidiana- que como se mencionó anteriormente, contiene varias manifestaciones a favor de la justicia y la igualdad de género, pero ofrece como recurso pedagógico un cuento especialmente misógino: "Carlita, la princesa que todo quería" (Ministerio de Educación Pública de Costa Rica, 2017a).

Carlita es descrita en dicha producción como una princesa caprichosa, manipuladora $y$ poco esforzada, que grita y patalea para obtener sus deseos (Ministerio de Educación Pública de Costa Rica, 2017a). Así, aunque explícitamente se diga que hay que respetar a las mujeres, el mensaje tácito del cuento es que las mujeres no serían tan respetables.

Esta idea de las mujeres como seres odiosos, se encontró insistentemente en el programa de Español sobre el cual se hablará, y da cuenta de la misoginia que se ha construido históricamente desde los saberes académicos y las iglesias. Tanto la filósofa Celia Amorós (2014) como Amelia Valcárcel (1997) han demostrado la existencia de una larga tradición misógina constituida en un proyecto político, con origen en el Romanticismo, que ubica a las mujeres como idénticas a sí mismas, por tanto, indiferenciadas e indiferenciables,es decir, generalizables (Amoros, 2014; Valcárcel, 1997). A partir de lo anterior se posibilita que, por decir algo, la actitud caprichosa y manipuladora de la infantilizada Carlita sea algo que el sentido común atribuye a todas las mujeres.

La presentación de las mujeres como seres llenos de defectos es fundamental en el andamiaje cultural, pues facilita que todo lo deleznable que les ocurre sea interpretado como algo natural (Valcárcel, 1997), es decir, normal e inevitable.

En el mismo sentido, el programa de Educación Física ${ }^{6}$ incluye en contenidos curriculares el "Respeto por la diversidad de género, evidenciado en el establecimiento de relaciones sanas entre varón-niña" (Ministerio de Educación
Pública de Costa Rica, 2013a, p.37). Al mismo tiempo, convoca a la integración de hombres y mujeres como medio para estimular una convivencia de paz y de construcción que se sugiere interpretar como un llamado al orden frente al "desorden" que provendrían de los discursos liberales. Si así fuera, lo que parece moderno, el respeto a la diversidad de género, resulta conservador pues es una diversidad que admite únicamente mujeres $y$ hombres.

Se observa con algún detalle los otros programas, empezando con el de Español ${ }^{7}$, en el que se habla de la diversidad y del respeto hacia el estudiantado, al tiempo que se plantean didácticamente las convenciones sociales necesarias para "expresar normas de caballerosidad" (Ministerio de Educación Pública de Costa Rica, 2013b). Ese llamado a la caballerosidad reafirma su sentido profundamente conservador cuando se repara en la literatura usada, a lo largo del documento, como recurso pedagógico.

Por un lado, está "El robot enamorado", que trata de eso, de un robot que tenía "su ruidito seductor de maquinaria vieja y herrumbrada" (Ministerio de Educación Pública de Costa Rica, 2013b) y que "se aproximó coqueteando $y$ se detuvo frente a una niña de pelito crespo que se le quedó mirando burlona" (Ministerio de Educación Pública de Costa Rica, 2013b, pp. 273). Es importante detenerse aquí, primeramente por el hecho de atribuir a un robot — masculino- impulsividad sexual, con lo cual se está naturalizando lo que los hombres supuestamente son, incluso siendo máquinas; por otro lado, la niña vista como objeto sexual, representada además como desagradable. A continuación se analizará la siguiente cita:

Miró (robot) hacia a todos lados y vio un extraño personaje verde y lustroso que caminaba muy despacio por el parque ¡Esa dama sí era bonita! pequeña de caparazón dura igual a la de él y con el lento y misterioso caminar. Resorte sintió en su pancita un extraño cosquilleo y se volvió rosado como un

$7 \quad$ Segundo Ciclo.

$6 \quad$ Primero y Segundo Ciclos. 
amanecer. La tortuga parpadeó asombrada; él bailó su danza de carraco, ella sonrió coqueta; él le regaló su corazón (Ministerio de Educación Pública de Costa Rica, 2013b, p.274).

Nuevamente se evidencia - en esta ocasión en un animal- un comportamiento que se atribuye a las mujeres - la coqueteríacomo un mecanismo de confirmación del comportamiento de género, haciendo uso además de una sobregeneralización que no distingue especies, pues se considera que puede ser leído como si las tortugas son coquetas, como las mujeres.

En este mismo sentido, la fábula "El águila y los gallos", atribuida a Esopo, también refiere a una supuesta naturaleza sexual masculina al narrar cómo dos gallos se pelean para que las gallinas los elijan, cómo un gallo alardea frente a otro y lo empuja sacándolo del gallinero y cómo es raptado por una águila, con lo cual: "Desde entonces el gallo que había perdido la riña se quedó con todo el gallinero" (Ministerio de Educación Pública de Costa Rica, 2013b, p.295). Se sugiere que es factible interpretar de dicha fábula que el gallo/hombre - cuya sexualidad es compulsiva - se quedó con todas las gallinas/mujeres. Es decir, que trata de la cualidad intercambiable entre las mujeres como objeto de reparto entre los sujetos hombres (Irigaray, 2009), y la idea de que las mujeres son indistinguibles (Amorós, 2014), lo cual hace posible que un gallo las quiera a todas, no a una en especial, sino a todas.

Igualmente, el poema "Las gafas de la jirafa" retrata a una jirafa que no tiene buena vista $y$ cree que "un enorme pino era un jirafo muy fino que le iba a hacer la corte ¡Vaya corte!" (Ministerio de Educación Pública de Costa Rica, 2013b, p.304). Desde el punto de vista de este análisis, esta imagen contribuye a justificar el mito del interés sexual desbocado de los hombres hacia las mujeres, presentándolas a ellas bajo un deseo por ser perseguidas por un hombre. Esto es la idea de que, en el fondo, las mujeres, aunque digan que no, están diciendo que sí, con lo que se justifica los avances sexuales - violentos o no-de los hombres.
Una idea tan extendida que ha sido enfrentada recientemente mediante consignas, tales como: "no es no"; campañas como \#\#NoesNo\# de los ayuntamientos de toda España; canciones como "No es No" del grupo Penadas por la Ley; e imágenes feministas usadas en las marchas y en las diversas protestas.

En esta dirección, "El patito feo" narra cómo la mamá pata se lamenta de que el papá pato la abandonara a ella y a sus patitos, al tiempo que celebra que el patito que le salió feo sea macho "De todos modos, es macho y no importa tanto - añadió-. Estoy segura de que será muy fuerte y se abrirá camino en la vida" (Ministerio de Educación Pública de Costa Rica, 2013b, p. 329).

Al mismo tiempo, los patos de otras bandadas se previenen "¡Eres más feo que un espantapájaros! - dijeron los patos salvajes. -Pero eso no importa, con tal que no quieras casarte con una de nuestras hermanas... No muy lejos, en otro pantano, viven una gansitas salvajes muy presentables, todas solteras, que saben graznar espléndidamente" (Ministerio de Educación Pública de Costa Rica, 2013b, p.330). Con estos contenidos, además de difundir y preservar la idea de que en las mujeres lo importante es la belleza física (para atraer marido), hay en este texto una sexualización muy explícita: las gansas que son salvajes, presentables, solteras y buenas entertainers.

Representar a la especie femenina de esa forma, además de ser moralmente objetable y malintencionado, acarrea consecuencias con las que las mujeres deberán lidiar, tales como, los perjuicios laborales. Por ejemplo, María Flórez-Estrada encontró en una investigación que realizó en tres empresas (una productora de software, otra "contact center" y una de manufactura de componentes electrónicos), que había una segregación sexual: los hombres en ocupaciones técnicas y científicas, mientras que las mujeres en áreas sociales y administrativas. Además, las ocupaciones mayormente llevadas a cabo por hombres eran mejor pagadas. Asimismo, las mujeres estaban constreñidas por su disposición a maternar y "levantar" a los hombres, a ser tímidas y modestas con sus logros, lo que ocasionaba que más que ser team 
leaders, lo cual les daría mayores oportunidades de ascenso y aumentos salariales, las mujeres tendían a ocupar el lugar de cheer leaders (Flórez-Estrada, 2007).

Se suma a lo afirmado que, en los recursos literarios anteriores, hay referencias sexuales claramente establecidas, cuyo objetivo será enseñar a las niñas a ser mujeres $y$ a los niños a ser hombres mediante la imitación y la reproducción del amor romántico (Esteba, 2011; Valcárcel, 1997).

Este programa usa otros cuentos como recurso para la formación que se agrupan para un fin muy específico: el de transmitir un "estate quieta" a las mujeres quienes son representadas por lo demás, como necesitadas de una guía moral. El primero se trata de la "Rosa caprichosa y los animales del jardín" que describe a una niña que pedía todo lo que quería $y$ aprende a pedir solo lo necesario, a cambio se ve recompensada por el hecho de que nadie más continuó diciéndole caprichosa (Ministerio de Educación Pública de Costa Rica, 2013b).

También el cuento "La brujita dulce" sobre una bruja que era buena en el fondo, pero sufría porque no sabía cómo serlo, debido a que estaba rodeada de brujas malas que la obligaban a serlo, hasta que unos niños le enseñaron a ser buena mediante un camino específico "que lo único que había que hacer era ayudar a los demás y hacer cosas por ellos" (Ministerio de Educación Pública de Costa Rica, 2013b, p. 277). Como se observa, en ambos relatos las protagonistas tratan de ser, dar y tener, bajo una clasificación de las mujeres en dos categorías morales que no deja mucha elección: o rosa o bruja.

Esta clasificación según la cual las mujeres o están del lado de la bondad o de la maldad, tiene un origen judeocristiano $y$ ha sido construido como un arquetipo, según han estudiado teóricas como Federici (2010). En esa línea, como en el cuento "Caperucita Roja"8, usado en este programa (Ministerio de Educación Pública de Costa Rica, 2013b), se deja una lección moral de obediencia so pena de no evitar el peligroso castigo.
El programa contiene otro cuento "La rosa pretenciosa"9 que detalla una de las posibilidades morales de las mujeres: la maldad. Este dice que "Erase una vez rosa muy coqueta $y$ vanidosa, que como veía que todos se detenían ante ella para alabar su belleza, ni siquiera quería hablarles a las otras flores del jardín". Ante su actitud, una abeja tuvo que intervenir para sentenciarla diciéndole que, si bien era la más hermosa, no era la más dulce $y$, como resultado de la debilidad moral de la rosa, es castigada con la pena de muerte: la cortan y la ponen en un jarrón para que cumpla una función que le critican implícitamente: exhibirse (Ministerio de Educación Pública de Costa Rica, 2013b, p. 368).

El mensaje de este último cuento es muy truculento y esquizofrénico porque a las mujeres sí se las manda a ser bellas, como se mencionó antes, pero serían los hombres quienes se encargan de hacer valer $y$ notar dicha belleza, no las mujeres en sí mismas.

Por otro lado, los programas de educación religiosa ${ }^{10}$ son profundamente conservadores y establecen una relación directa entre la sexualidad (a la que siempre adjuntan los adjetivos de humana, cristiana, integral, afectiva y comprometida personal y socialmente) y el afecto, dándole un carácter deserotizado y atribuyéndole el papel de "pegamento comunal". Por lo demás, abundan en sus declaraciones la importancia de que las diferencias y las semejanzas entre sexos se hagan efectivas mediante un mecanismo de complementación (Ministerio de Educación Pública de Costa Rica, 2005).

Para finalizar, se recalca el hecho de que los cuatro cuentos llaman a las mujeres a conformarse con poco, a servir y aceptar tutela, como lo estableció el derecho romano para todas aquellas que no estuvieran en el grupo de vírgenes vestales (Pérez, 2017). Sencillas en sus ambiciones, serviciales e incapaces de valerse por sí mismas, el futuro más atractivo sería el tener marido.

\footnotetext{
$9 \quad$ De Ernesto Langer.

10 Segundo Ciclo.
}

$8 \quad$ Versión de los hermanos Grimm. 


\section{REFLEXIONES TEMPORALES}

Primeramente, se desea insistir en que los programas que se analizaron en esta investigación, en comparación con los anteriores, muestran una mejoría relativa en tanto no se nota el mismo ensañamiento contra la variedad sexual y de género, sin embargo, son más agresivos contra las mujeres.

Son programas de extremos, como si hubieran sido elaborados por dos equipos diferentes $y$ con pensamientos opuestos, uno marcadamente liberal $y$ otro tradicional. $\mathrm{O}$ como si fueran producto de una negociación, en este caso, con la Iglesia Católica, la cual integra varias comisiones del MEP. Debido a esto, el ala liberal acepta contenidos conservadores en unos textos y el ala conservadora hace lo mismo con los postulados liberales para unos programas.

Otro elemento que llama la atención es que, con cierta frecuencia, se alude al respeto a la diversidad, ya sea producto de una discapacidad, de vivencias culturales, religiosas, étnicas, familiares o económicas, pero sin mencionar la diversidad sexual que queda en una especie de paréntesis, en unos puntos suspensivos o en un etcétera.

\section{REFERENCIAS}

Amorós, C. (2014). Salomon no era sabio. Ed. Fundamentos.

Butler, J. (2011). Gender Trouble: Feminism and the Subversion of Identity Routledge. http://lauragonzalez.com/TC/ BUTLER_gender_trouble.pdf

Bourdieu, P. (1999). Meditaciones pascalianas. Anagrama.

Bourdieu, P. y Passeron, J. (1995). La reproducción: Elementos para una teoría del sistema de enseñanza. Fontamara. https://socioeducacion.files.wordpress. com/2011/05/bourdieu-pierre-la-reproduccion1.pdf

Calvo, Y. (2017). De Mujeres, palabras y alfileres. Ed. Bellasterra.

Clasificación Internacional de Enfermedades. (2019). Estandarización mundial de la información de diagnóstico en el ámbito de la salud. https://icd.who.int/ browse11/1-m/es
Corte Interamericana de Derechos Humanos. (2017). La República de Costa Rica Identidad de género, e igualdad y no discriminación a parejas del mismo sexo. Opinión Consultiva OC-24/17 de 24 de noviembre. http://www.corteidh.or.cr/ docs/opiniones/seriea_24_esp.pdf

Esteba, M. (2011): Crítica del pensamiento amoroso: Temas contemporáneos. Ed. Bellaterra.

Federici, S. (2010). Calibán y la bruja: Mujeres, cuerpo y acumulación primitiva. Traficantes.

Flórez-Estrada, M. (2007). Economía del género: el valor simbólico y económico de las mujeres. Ed. UCR.

Flórez-Estrada, M. (2009). La construcción social de significados en el fin de la era del Padre. Revista de Ciencias Sociales, 125, 117-125.

Flórez-Estrada, M. (2019). Identidades sexuadas modernas costarricenses (18331930): de la sociedad viva a la comunidad cerrada. Cuadernos Inter.c.a.mbio sobre Centroamérica y el Caribe, 16(2). Universidad de Costa Rica.

Flores, A. y Espejel, A. (2015). El sexismo como una práctica de violencia en la Universidad. RES, Revista de Educación Social, (21), 128-142.

Foucault, M. (2002). Vigilar y castigar. Nacimiento de la prisión. Siglo veintiuno ed.

Gamboa, I. (2009). En el Hospital Psiquiátrico, el sexo como locura. Ed. UCR.

Gamboa, I. (2010). Sexualidad en la primaria, tratado de un inútil combate. Revista de Historia, 61-62, 115-147.

Guzmán, V. y Bonan, C. (2007). Feminismo y modernidad. Debate Feminista, 35, 257-274.

Irigaray, L. (2009). Ese sexo que no es uno. Ed. Akal.

Keller, R. (2011). The Sociology of Knowledge Approach to Discourse (SKAD). Human Studies 34(1), 43-65.

Laqueur, T. (2007). Sexo solitario: Una historia cultural de la masturbación. Fondo de Cultura Económica. 
Mesa, S. (2019). Carreras académicas de mujeres en la Universidad de Costa Rica: Un reto para la igualdad. Revista Electrónica "Actualidades Investigativas en Educación, 19(1). https://revistas.ucr. ac.cr/index.php/aie/article/view/35716

Ministerio de Educación Pública de Costa Rica. (2005). Programas de Estudios Educación Religiosa segundo ciclo. San José. https://www.mep.go.cr/sites/default/ files/programadeestudio/programas/educreligiosa2ciclo-2005.pdf

Ministerio de Educación Pública de Costa Rica. (2013a). Programas de Estudio de Educación Física: primero y segundo Ciclos de la Educación General Básica. San José. https://www.mep.go.cr/sites/ default/files/programadeestudio/programas/efisicaly2ciclo.pdf

Ministerio de Educación Pública de Costa Rica. (2013b). Programa de estudio de Español Segundo Ciclo de la Educación General Básica. San José. https://www.mep.go.cr/ sites/default/files/programadeestudio/ programas/espanol2ciclo.pdf

Ministerio de Educación Pública de Costa Rica. (2014). Plan de estudios Centro Nacional de Educación Helen Keller. San José. https://www.mep.go.cr/sites/default/files/ programadeestudio/programas/eespecial_hellen_keller.pdf

Ministerio de Educación Pública de Costa Rica. (2016). Programas de Estudio de Inglés: segundo ciclo. San José. https://www. mep.go.cr/sites/default/files/programadeestudio/programas/ingles_2ciclo.pdf

Ministerio de Educación Pública de Costa Rica. (2017a). Programa de Estudio de Educación para la Vida Cotidiana: Primero y Segundo Ciclos de Educación
General Básica. San José. https://www. mep.go.cr/sites/default/files/programadeestudio/programas/vida-cotidianaly2ciclos.pdf

Ministerio de Educación Pública de Costa Rica. (2017b). Programas de estudio de Orientación Primero, segundo y Tercer ciclos de la Educación General Básica y Educación Diversificada. San José. https://www.mep.go.cr/sites/default/files/ programadeestudio/programas/orientacion-nuevo.pdf

Pérez, V. (2017). Capacidad de la mujer en derecho privado Romano. Revista Clepsydra, (16), 191-217. https://dialnet.unirioja.es/ servlet/articulo?codigo $=6267921$

Programa de Naciones Unidas para el Desarrollo-PNuD. (2019). Informe Nacional de Desarrollo Humano. San José, Costa Rica. http://hdr.undp.org/ sites/default/files/hdr_2019_overview_-spanish.pdf

Preciado, P. (2013). Testo Junkie: sex, drugs, and biopolitics in the pharmacopornographic era. The Feminist Press at the City University of New York.

Valcárcel, A. (1997). La política de las mujeres. Ed. Cátedra.

Van Dijk, T. (2018). Discourse and Migration. En R. Zapata-Barrero y E. Yalaz (Eds.), Qualitative Research in European Migration Studies (pp. 227-246). https://www.researchgate.net/publication/325867494_Discourse_and_ Migration

Fecha de ingreso: 24/02/2020 Fecha de aprobación: 14/10/2020 